\title{
ESTOQUES DE BIOMASSA E CARBONO EM UNIDADE DE CONSERVAÇÃO NO BIOMA MATA ATLÂNTICA
}

\author{
BIOMASS AND CARBON STOCKS IN A CONSERVATION UNIT IN THE BIOMA MATA \\ ATLÂNTICA Miguel Nascimento ${ }^{4}$, Danilo Henrique dos Santos Ataíde ${ }^{5}$, Vinícius Augusto Morais ${ }^{6}$
1, 2, 4, 5 Universidade Federal Rural do Rio de Janeiro (UFRRJ), Seropédica, Rio de Janeiro, Brasil - laiscandido30@hotmail.com, ejgaraujo@gmail.com, alexmnasci@gmail.com\& \\ Laís Cândido Silva', Emanuel José Gomes de Araújo ${ }^{2}$, Rafaella de Angeli Curto ${ }^{3}$, Alexandre \\ daniloataide.florestal@gmail.com \\ ${ }^{3}$ Universidade Federal de Mato Grosso, Instituto de Ciências Agrárias e Ambientais, Sinop, Mato Grosso, \\ Brasil-rafaellacurto@yahoo.com.br \\ ${ }^{6}$ Universidade Estadual de Mato Grosso, Alta Floresta, Mato Grosso, Brasil - vemorais@gmail.com
}

\section{RESUMO}

Diante da intensificação das emissões de gases do efeito estufa, as formações florestais possuem papel importante na fixação do dióxido de carbono $\left(\mathrm{CO}_{2}\right)$ da atmosfera. Diferentes espécies, famílias e grupos ecológicos contribuem de forma distinta no potencial de estoque de carbono. Com isso, objetivou-se nesse trabalho estimar a biomassa e o carbono estocado em espécies florestais presentes em uma Unidade de Conservação no bioma Mata Atlântica. Foram alocados 40 pontos quadrantes no Parque Natural Municipal do Curió, Paracambi, RJ, sendo mensuradas as variáveis DAP e altura total das árvores pertencentes a cada ponto, além da coleta de baguetas via sonda de Pressler, nas alturas 0,70; 1,30 e 1,80 m do solo. Caracterizou-se a estrutura horizontal, definindo as espécies de maior valor de importância e seus grupos ecológicos. A biomassa foi quantificada e o estoque de carbono (EC) foi estimado ( $50 \%$ da biomassa). O $\mathrm{CO}_{2}$ equivalente estocado foi obtido pelo produto entre o fator 3,67 e o EC. Como resultado, foram mensuradas 160 árvores e identificadas 57 espécies. Os estoques de biomassa e carbono estimados foram 212,39 e 106,19 t.ha-1 respectivamente, sendo o grupo das espécies secundárias responsável por $38,95 \%$ desse estoque. A espécie com maior representatividade na fixação do $\mathrm{CO}_{2}$ foi Plathymenia reticulata Benth. $\mathrm{O} \mathrm{CO}_{2}$ equivalente estocado no trecho estudado totaliza 3.893,81 toneladas. A imobilização de $\mathrm{CO}_{2}$ pelas Unidades de Conservação corresponde a uma alternativa em projetos de Pagamentos por Serviços Ambientais, contribuindo para a manutenção dessas áreas e conservação da biodiversidade.

PALAVRAS-CHAVE: Gás carbônico, Grupo ecológico, Sonda de Pressler.

\section{ABSTRACT}

Faced with the intensification of greenhouse gas emissions, forest formations play an important role in setting carbon dioxide $\left(\mathrm{CO}_{2}\right)$ in the atmosphere. Different species, families and ecological groups contribute distinctly to the carbon stock potential. The objective of this study was to estimate the biomass and carbon stored in forest species present in a Conservation Unit in the Atlantic Forest biome. Forty-four points were allocated in the Curió Municipal Natural Park, Paracambi, RJ, and the variables DAP and total height of the trees belonging to each point were measured, as well as the collection of baguettes through Pressler borer, at heights of $0.70 ; 1.30$ and $1.80 \mathrm{~m}$ from the ground. The horizontal structure was characterized, defining the most important species of importance and their ecological groups. The biomass was quantified and the carbon stock (CE) was estimated ( $50 \%$ of the biomass). The $\mathrm{CO}_{2}$ equivalent stock was obtained by the product between factor 3.67 and EC. As a result, 160 trees were measured and 57 species identified. Estimated biomass and carbon stocks were 212.39 and 106.19 t.ha-1 respectively, and the secondary species group accounted for $38.95 \%$ of this stock. The most representative species in $\mathrm{CO}_{2}$ fixation was Plathymenia reticulata Benth. The $\mathrm{CO}_{2}$ equivalent stored in the studied section totals $3,893.81$ tons. The immobilization of $\mathrm{CO}_{2}$ by Conservation Units corresponds to an alternative in Payments for Environmental Services projects, contributing to the maintenance of these areas and conservation of biodiversity.

KEYWORDS: Carbon dioxide, Ecological group, Pressler borer. 


\section{INTRODUÇÃO}

O cenário de alterações climáticas pode ser classificado como um dos grandes desafios da atualidade. Um dos agentes causadores mais debatidos $\mathrm{e}$ evidenciados sobre o tema é a intensificação das emissões de gases do efeito estufa (GEE), como o dióxido de carbono $\left(\mathrm{CO}_{2}\right)$ originado pelas atividades humanas, por meio da queima de combustíveis fósseis e desmatamentos seguidos de queima do material vegetal rico em carbono orgânico (MORAIS et al., 2017).

Nesse sentido, as formações florestais possuem papel importante na fixação do $\mathrm{CO}_{2}$ da atmosfera, uma vez que este é requerido no processo fotossintético da planta, incorporando-o em biomassa, folhas e produtos metabólicos (ROCHADELLI, 2001). Com isso, as plantas apresentam contribuição direta para amenização da emissão de gases e fortalecem a importância da quantificação do estoque de carbono imobilizado em florestas naturais.

Esse fato amplia a ênfase nos últimos anos para o papel das florestas naturais na captura de $\mathrm{CO}_{2}$, corroborado pela avaliação do estoque de carbono em formações de Floresta Ombrófila Densa (CUNHA et al., 2009; SILVEIRA, 2010) e Mista (WEBER et al., 2005; AMARAL et al., 2010; WATZLAWICK et al., 2012; MOGNON et al., 2013; LIPINSKI et al., 2017;), Floresta Estacional Semidecidual (RIBEIRO et al., 2009; SOUZA et al., 2011; AMARO et al., 2013; DINIZ et al., 2015;) e biomas como Cerrado (MORAIS et al., 2013) e Caatinga (SANTOS et al., 2016).

Com relação à importância das formações florestais, as Unidades de Conservação (UCS) são áreas de extrema importância na conservação da biodiversidade e manutenção do meio natural, manutenção do patrimônio genético e proteção dos ecossistemas (HASSLER, 2005). Assim, elas constituem uma importante área de estudo sobre a dinâmica natural da biomassa e as relações existentes entre a floresta e a fixação do $\mathrm{CO}_{2}$ atmosférico, tanto em captura quanto em imobilização.

Moreira-Burger \& Delitti (1999) acreditam que a biomassa tem um importante papel na caracterização estrutural dos ecossistemas. Os autores a caracterizam como um fator de grande relevância na análise e monitoramento dos ecossistemas, na avaliação de impactos, na análise de estágios de sucessão e na avaliação de produtividade primária de uma formação vegetal.

A ausência de estudos para quantificação da biomassa e fixação de carbono se deve a grande dificuldade de estimativas precisas sobre a contribuição de cada indivíduo ou população nos ecossistemas, ao oneroso trabalho demandado nessas análises, à dificuldade de coleta de dados de campo, principalmente na utilização de métodos destrutivos, à complexidade de compreensão dos fenômenos ligados a fixação de carbono e ao fato desse tema ainda ser relativamente recente (MOREIRABURGER \& DELITTI, 1999; SANQUETTA et al., 2003; WEBER et al., 2005; ARAÚJO et al., 2017).

A demanda por análises adequadas, a busca por custos reduzidos, a procura por ganho de confiabilidade nas estimativas e o crescimento pelo mercado de créditos de carbono e redução das alterações climáticas (NICOLETTI et al., 2015), suscita uma demanda muito importante por estudos sobre a fixação de carbono na biomassa florestal.

Em virtude disso, é importante buscar informações acerca das diferentes contribuições no potencial de estoque de carbono de que espécies florestais, famílias e grupos ecológicos distintos apresentam. Assim, objetivouse com o presente estudo, estimar a biomassa e o carbono estocado em espécies florestais presentes em Unidade de Conservação no bioma Mata Atlântica.

\section{MATERIAL E MÉTODOS}

\section{Área de estudo}

Os dados foram coletados em uma área de aproximadamente 10 ha, com coordenadas centrais $22^{\circ} 35^{\prime} 53^{\prime \prime} \mathrm{S}, 43^{\circ} 42^{\prime} 15^{\prime \prime} \mathrm{O}$, no Parque Natural Municipal do Curió (PNMC), município de Paracambi, RJ (Figura 1). 0 PNMC é uma Unidade de Conservação caracterizada por alta diversidade biológica, com vegetação característica de Floresta Ombrófila Densa, majoritariamente com formação secundária (SMMAP \& ITPA, 2010).

O parque está inserido na Bacia Hidrográfica do Rio Guandu, com clima predominante do tipo Aw, segundo a classificação de Köeppen (ALVARES et al., 2014), variando de brando subtropical a tropical quente/úmido. A altitude varia de 100 e $690 \mathrm{~m}$; com temperatura e precipitação médias de $23,4^{\circ} \mathrm{C}$ e $1.050 \mathrm{~mm}$, respectivamente (FRAGA et al., 2012). De acordo com Teixeira et al. (2014), a vegetação do PNMC apresenta-se em regeneração natural, sem intervenções, por aproximadamente 60 anos. 


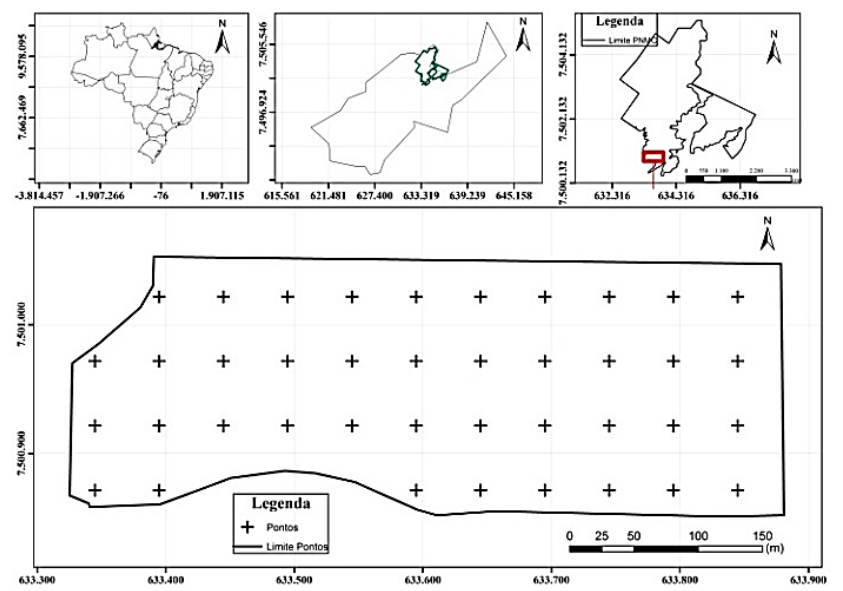

Figura 1. Área amostrada no PNMC, Paracambi, RJ.

\section{Coleta de dados}

Os dados foram coletados em 40 unidades amostrais, por meio do processo de amostragem sistemática, alocadas pelo método de área variável de pontos quadrantes (COTTAM \& CURTIS, 1956). Considerou-se sempre o eixo das ordenadas no sentido Norte-Sul. A intensidade amostral foi de um ponto a cada $2.500 \mathrm{~m}^{2}$.

Em cada quadrante foram mensuradas as variáveis diâmetro a 1,3 $\mathrm{m}$ do solo $(D A P)$ e a altura total da árvore mais próxima da origem do ponto amostral, com DAP $\geq$ $5,0 \mathrm{~cm}$. Também foi obtida a distância de cada árvore até a origem do plano cartesiano, além de seu georreferenciamento, identificação botânica conforme o sistema APG III (2009) e classificação por família e grupo ecológico.

Para a determinação do teor de carbono foi realizada a coleta de amostras de madeira do fuste, utilizando trado de incremento (sonda de Pressler), sendo as amostras coletadas nas alturas de 0,7; 1,3 e 1,8 m do solo. Essas amostras foram dispostas em sacos plásticos, devidamente identificadas, acondicionadas em recipiente térmico a fim de evitar a perda da umidade e, por fim, encaminhadas ao Laboratório de Mensuração e Manejo Florestal da Universidade Federal Rural do Rio de Janeiro.

\section{Análise de Dados}

Para descrever o estágio sucessional da vegetação, foram obtidos os valores médios de DAP, altura total e de área basal. Também foram estimados os índices (Densidade, Dominância e Frequência), absolutos e relativos, para retratar a estrutura horizontal da floresta, e definir as espécies de maior índice de valor de importância (IVI).

A riqueza florística da área foi estimada pelos índices de diversidade de Shannon $\left(H^{\prime}\right)$, Simpson (S) e Quociente de Mistura de Jentsch (QM), além da Equabilidade de Pielou (J'), conforme descritos em Scolforo et al. (2008).

Para caracterizar a estrutura diamétrica, obteve-se a distribuição de frequência dos valores de DAP, agrupados em 9 classes com amplitude de $10 \mathrm{~cm}$, definidas empiricamente. A biomassa e o carbono estocados foram determinados a partir do volume verde de cada amostra conforme demonstrado:

$$
V_{\text {verde }}=\frac{d^{2} * l * \pi}{4}
$$

Em que: $V_{\text {verde }}=$ volume verde $\left(\mathrm{cm}^{3}\right) ; d=$ diâmetro da amostra de madeira $(\mathrm{cm})$; e $l=$ comprimento da amostra de madeira $(\mathrm{cm})$.

As amostras foram pesadas com teor de umidade acima de $30 \%$ e posteriormente acondicionadas em estufa para secagem a $103 \pm 2 \stackrel{\circ}{ } \mathrm{C}$, por 48 horas. Após a secagem, as amostras foram novamente pesadas para obtenção do peso seco (PS, em g). A densidade básica da amostra foi determinada pelo estimador:

$$
D_{b}=\frac{P S}{V_{\text {verde }}}
$$

Em que: $D_{b}=$ densidade básica $\left(\mathrm{kg} \cdot \mathrm{m}^{-3}\right) ; P S=$ peso seco $(\mathrm{kg}) ; V_{\text {verde }}=$ volume verde $\left(\mathrm{cm}^{3}\right)$.

A densidade média da madeira $\left(\bar{d}_{b}\right)$, em $\mathrm{kg} \cdot \mathrm{m}^{-3}$, para cada árvore, foi obtida por meio da média das densidades das três amostras coletadas por árvore.

O volume total com casca de cada árvore, foi obtido por meio da Equação proposta por Scolforo et al. (2008), com $R^{2}=99,27$ e $S_{y x}(\%)=23,24$.

$\operatorname{Ln}($ VTCC $)=-9,975249+[2,171914 \cdot \operatorname{Ln}(D A P)]+[1,147984 \cdot \operatorname{Ln}(H)]$

Em que: $V T c c=$ volume total com casca $\left(\mathrm{m}^{3}\right) ; D A P=$ diâmetro a 1,3 $\mathrm{m}$ do solo $(\mathrm{cm})$; e $H=$ altura total da árvore $(\mathrm{m})$.

A biomassa e o estoque de carbono em cada árvore foram estimados por meio das seguintes equações:

$$
\begin{aligned}
& B=\bar{d}_{b} \cdot V T_{c c} \\
& E C=0,5 \cdot B
\end{aligned}
$$

Em que: $B=$ biomassa $(\mathrm{kg}) ; \bar{d}_{b}=$ densidade média da madeira (kg. $\mathrm{m}^{-3)} ; V T_{c c}=$ Volume total com casca $\left(\mathrm{m}^{3}\right)$; e $E C=$ estoque de carbono $(\mathrm{kg})$. 
Em sequência, estimou-se o Gás Carbônico equivalente estocado em cada árvore por meio da expressão:

$$
\mathrm{CO}_{2}=3,67 \cdot E C
$$

Em que: $\mathrm{CO}_{2}=$ dióxido de carbono equivalente $(\mathrm{t}) ; 3,67=$ fator obtido pela razão entre a massa molecular do Gás Carbônico (igual a 44) e a massa atômica do carbono (igual a 12), de acordo com Brown et al. (1986) e Hoen \& Solberg (1994); e $E C$ = estoque de carbono (kg).

Por fim, foram mensuradas as médias das variáveis de interesse por árvore e por espécie, a fim de identificar suas contribuições médias no valor total da floresta. Além disso, foi realizado o agrupamento dos indivíduos identificados quanto à espécie e quanto ao grupo ecológico pertencente visando à estratificação dos dados e consequentemente melhoria nas inferências quanto aos parâmetros analisados.

\section{RESULTADOS E DISCUSSÃO}

Com a metodologia utilizada pelo presente estudo, foram mensuradas 160 árvores, nos 40 pontos quadrantes, contabilizando 57 espécies, 47 gêneros e 30 famílias. A Equabilidade de Pielou ( $\left.J^{\prime}\right)$ foi igual a 0,87, indicando que existe uniformidade de distribuição dos indivíduos nas espécies. Valor muito próximo ao encontrado por Cysneiros et al. (2015), que em seu estudo verificou um índice J' de 0,88 em área próxima no PNMC. O índice de Diversidade de Shannon e de Simpson foram 3,50 e 0,04 respectivamente, e o quociente de mistura de Jentsch foi de 0,36.

A Diversidade de Shannon encontrada expressa uma diversidade comumente alta para floresta secundária, o que pode ser entendido pela presença de fragmentos primários próximos ao local e pelo histórico de recuperação da área. Além disso, a floresta encontra-se em estágio médio de regeneração, agregando consigo maior diversidade. Esses valores encontrados corroboram com os descritos por Teixeira et al. (2014), na mesma Unidade de Conservação, cuja variação foi de 3,19; 3,63 e 3,72 para os três sítios analisados em seu trabalho, porém foram menores do que o citado por Cysneiros et al. (2015), que obteve o valor de 4,7 para o mesmo parque.

Concomitantemente, a estrutura diamétrica estabelecida estratificou os indivíduos em 9 classes de diâmetro, apresentando tendência exponencial negativa. O maior número de árvores (114) ocorreu na menor classe de diâmetro, com intervalo variando de 5 a $15 \mathrm{~cm}$ (Figura 2).

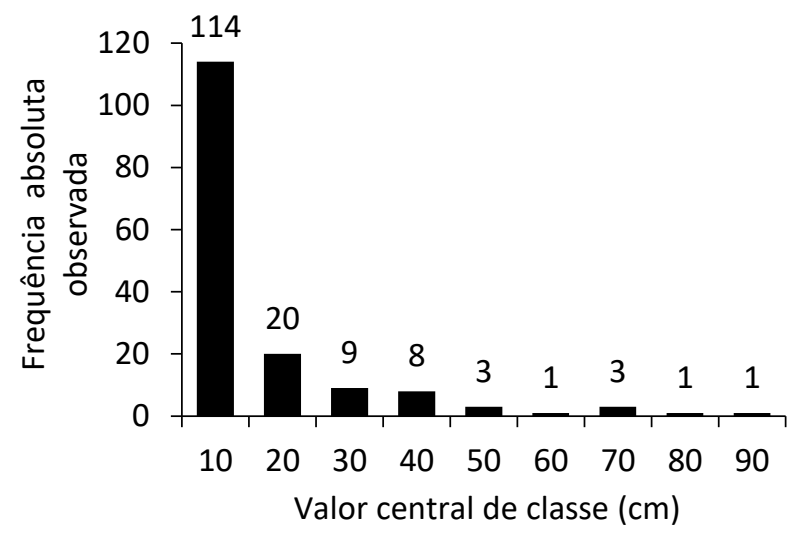

Figura 2. Estrutura diamétrica do PNMC, Paracambi, RJ.

A floresta estudada apresenta-se em estágio médio de sucessão ecológica, segundo a resolução CONAMA $\mathrm{N}^{\circ}$ 006/94. O DAP médio é $15,7 \mathrm{~cm}$ apresentando área basal de 23,9 $\mathrm{m}^{2}$.ha ${ }^{-1}$ e a altura média é 9,66 m. Foram observadas espécies características de vegetação nesse estágio como Guarea guidonia (L.) Sleumer (Meliaceae) e Cupania oblongifolia Mart. (Sapindaceae). Entretanto, o local apresenta ainda espécies de características iniciais a avançadas de sucessão como Trema micrantha (L.) Blume, Ocotea sp., Nectandra sp., Joannesia princeps Vell., Cariniana legalis (Mart.) Kuntze e Gallesia integrifolia (Spreng.) Harms (RESOLUÇÃO CONAMA no 006, 1994), além da presença de espécies exóticas, como Artocarpus heterophyllus Lam.

A maior distribuição das espécies na classe definida como secundária inicial (41\%) indica a transição de estágios iniciais para estágios mais avançados de sucessão ecológica após todo o processo de ocupação e regeneração ocorrido na área.

Teixeira et al. (2014) verificaram na mesma área, avaliando a regeneração da floresta em três sítios amostrais sob diferentes níveis de perturbação (controle, moderadamente perturbado e perturbado), o predomínio do grupo de secundárias tardias, com $56 \%$, 52\% e $44 \%$ do total de 233 indivíduos amostrados. Isso pode ser compreendido pelo alto grau de perturbação sofrido na área de amostragem desse trabalho, uma vez que está situada no limite entre parque e cidade, possui uma das trilhas mais conhecidas e utilizadas e encontra-se muito próxima de zonas de captação e tratamento de água. Todas essas particularidades podem interferir no processo de recuperação natural da área. 
Tabela 1. Lista de espécies encontradas no PNMC, Paracambi, RJ, classificadas segundo o Índice de Valor de Importância.

\begin{tabular}{|c|c|c|c|c|c|c|c|c|c|c|c|c|c|c|}
\hline Espécies & GE & DA & DR & FA & FR & DoA & DoR & IVI & B (t.ha-1) & C (t.ha-1) & $\mathrm{CO}_{2}\left(\right.$ t.ha $\left.^{-1}\right)$ & $B\left(\right.$ t.arv $\left.{ }^{-1}\right)$ & $C\left(t . a^{-1}\right)$ & $\mathrm{CO}_{2}\left(\mathrm{t}_{\mathrm{arv}} \mathbf{a r}^{-1}\right)$ \\
\hline Guarea guidonia (L.) Sleumer & ST & 26,35 & 3,13 & 12,50 & 3,70 & 6,64 & 20,75 & 9,19 & 48,23 & 24,11 & 88,42 & 1,83 & 0,92 & 3,36 \\
\hline Artocarpus heterophyllus Lam. & $\mathrm{E}$ & 68,50 & 8,13 & 20,00 & 5,93 & 3,77 & 11,77 & 8,61 & 20,91 & 10,45 & 38,33 & 0,31 & 0,15 & 0,56 \\
\hline Cupania oblongifolia Mart. & $\mathrm{PI}$ & 47,42 & 5,63 & 17,50 & 5,19 & 1,67 & 5,21 & 5,34 & 11,09 & 5,54 & 20,33 & 0,23 & 0,12 & 0,43 \\
\hline Actinostemon verticillatus (Klotzsch) Baill. & $\mathrm{SI}$ & 57,96 & 6,88 & 22,50 & 6,67 & 0,54 & 1,70 & 5,08 & 1,54 & 0,77 & 2,82 & 0,03 & 0,01 & 0,05 \\
\hline Guapira opposita (Vell.) Reitz & $\mathrm{SI}$ & 52,69 & 6,25 & 25,00 & 7,41 & 0,49 & 1,54 & 5,07 & 1,20 & 0,60 & 2,20 & 0,02 & 0,01 & 0,04 \\
\hline Erythroxylum pulchrum A.St.-Hil. & SI & 36,88 & 4,38 & 15,00 & 4,44 & 0,61 & 1,90 & 3,57 & 2,95 & 1,48 & 5,42 & 0,08 & 0,04 & 0,15 \\
\hline Sorocea bonplandii (Baill.) W.C.Burguer et al. & SI & 36,88 & 4,38 & 15,00 & 4,44 & 0,17 & 0,52 & 3,11 & 0,32 & 0,16 & 0,58 & 0,01 & 0,00 & 0,02 \\
\hline Ceiba speciosa (A.St.-Hil.) Ravenna & $\mathrm{PI}$ & 10,54 & 1,25 & 5,00 & 1,48 & 2,09 & 6,55 & 3,09 & 7,84 & 3,92 & 14,37 & 0,74 & 0,37 & 1,36 \\
\hline Trichilia casaretti C.DC. & SI & 31,62 & 3,75 & 12,50 & 3,70 & 0,30 & 0,92 & 2,79 & 1,39 & 0,69 & 2,54 & 0,04 & 0,02 & 0,08 \\
\hline Senefeldera verticillata (Vell.) Croizat & ST & 26,35 & 3,13 & 12,50 & 3,70 & 0,26 & 0,80 & 2,54 & 0,97 & 0,48 & 1,77 & 0,04 & 0,02 & 0,07 \\
\hline Simira glaziovii (K.Schum.) Steyerm. & $\mathrm{SI}$ & 21,08 & 2,50 & 7,50 & 2,22 & 0,29 & 0,89 & 1,87 & 0,92 & 0,46 & 1,68 & 0,04 & 0,02 & 0,08 \\
\hline Heisteria perianthomega (Vell.) Sleumer & NC & 10,54 & 1,25 & 5,00 & 1,48 & 0,84 & 2,61 & 1,78 & 7,23 & 3,62 & 13,26 & 0,69 & 0,34 & 1,26 \\
\hline Ocotea sp. & NC & 21,08 & 2,50 & 7,50 & 2,22 & 0,13 & 0,42 & 1,71 & 0,53 & 0,27 & 0,98 & 0,03 & 0,01 & 0,05 \\
\hline Plathymenia reticulata Benth. & $\mathrm{PI}$ & 5,27 & 0,63 & 2,50 & 0,74 & 1,14 & 3,57 & 1,64 & 10,51 & 5,26 & 19,27 & 1,99 & 1,00 & 3,66 \\
\hline Joannesia princeps Vell. & SI & 15,81 & 1,88 & 7,50 & 2,22 & 0,12 & 0,38 & 1,49 & 0,17 & 0,08 & 0,30 & 0,01 & 0,01 & 0,02 \\
\hline Casearia sylvestris Sw. & $\mathrm{PI}$ & 15,81 & 1,88 & 7,50 & 2,22 & 0,09 & 0,28 & 1,46 & 0,24 & 0,12 & 0,45 & 0,02 & 0,01 & 0,03 \\
\hline Morfoespécie 6 & NC & 10,54 & 1,25 & 2,50 & 0,74 & 0,73 & 2,28 & 1,42 & 6,37 & 3,19 & 11,68 & 0,60 & 0,30 & 1,11 \\
\hline Astronium graveolens Jacq. & $\mathrm{SI}$ & 15,81 & 1,88 & 5,00 & 1,48 & 0,12 & 0,36 & 1,24 & 0,31 & 0,15 & 0,56 & 0,02 & 0,01 & 0,04 \\
\hline Morfoespécie 3 & NC & 5,27 & 0,63 & 2,50 & 0,74 & 0,54 & 1,70 & 1,02 & 4,22 & 2,11 & 7,74 & 0,80 & 0,40 & 1,47 \\
\hline Trichilia elegans A.Juss. & $\mathrm{SI}$ & 10,54 & 1,25 & 5,00 & 1,48 & 0,06 & 0,19 & 0,97 & 0,21 & 0,11 & 0,39 & 0,02 & 0,01 & 0,04 \\
\hline Sorocea hilarii Gaudich. & SI & 10,54 & 1,25 & 5,00 & 1,48 & 0,04 & 0,13 & 0,95 & 0,11 & 0,05 & 0,19 & 0,01 & 0,01 & 0,02 \\
\hline Morfoespécie 2 & NC & 5,27 & 0,63 & 2,50 & 0,74 & 0,44 & 1,39 & 0,92 & 2,47 & 1,23 & 4,53 & 0,47 & 0,23 & 0,86 \\
\hline Nectandra sp. & NC & 10,54 & 1,25 & 2,50 & 0,74 & 0,13 & 0,42 & 0,80 & 0,34 & 0,17 & 0,63 & 0,03 & 0,02 & 0,06 \\
\hline Sorocea sp. & NC & 10,54 & 1,25 & 2,50 & 0,74 & 0,11 & 0,34 & 0,78 & 0,26 & 0,13 & 0,47 & 0,02 & 0,01 & 0,04 \\
\hline Xylopia laevigata (Mart.) R.E.Fr. & $\mathrm{SI}$ & 5,27 & 0,63 & 2,50 & 0,74 & 0,21 & 0,65 & 0,67 & 1,60 & 0,80 & 2,93 & 0,30 & 0,15 & 0,56 \\
\hline Jacaratia spinosa (Aubl.) A.DC. & $\mathrm{SI}$ & 5,27 & 0,63 & 2,50 & 0,74 & 0,18 & 0,55 & 0,64 & 0,10 & 0,05 & 0,19 & 0,02 & 0,01 & 0,04 \\
\hline Quararibea turbinata (Sw.) Poir. & ST & 5,27 & 0,63 & 2,50 & 0,74 & 0,15 & 0,46 & 0,61 & 0,45 & 0,22 & 0,82 & 0,09 & 0,04 & 0,16 \\
\hline Myrcia splendens (Sw.) DC. & $\mathrm{PI}$ & 5,27 & 0,63 & 2,50 & 0,74 & 0,10 & 0,31 & 0,56 & 0,59 & 0,30 & 1,08 & 0,11 & 0,06 & 0,21 \\
\hline Hymenaea courbaril L. & $\mathrm{PI}$ & 5,27 & 0,63 & 2,50 & 0,74 & 0,09 & 0,29 & 0,55 & 0,58 & 0,29 & 1,06 & 0,11 & 0,05 & 0,20 \\
\hline Pseudopiptadenia contorta (DC.) G.P.Lewis \& M.P.Lima & SI & 5,27 & 0,63 & 2,50 & 0,74 & 0,09 & 0,27 & 0,54 & 0,15 & 0,08 & 0,28 & 0,03 & 0,01 & 0,05 \\
\hline
\end{tabular}




\begin{tabular}{|c|c|c|c|c|c|c|c|c|c|c|c|c|c|c|}
\hline Piptadenia gonoacantha (Mart.) J.F.Macbr. & $\mathrm{PI}$ & 5,27 & 0,63 & 2,50 & 0,74 & 0,08 & 0,25 & 0,54 & 0,29 & 0,14 & 0,53 & 0,05 & 0,03 & 0,10 \\
\hline Morfoespécie 4 & NC & 5,27 & 0,63 & 2,50 & 0,74 & 0,07 & 0,21 & 0,53 & 0,33 & 0,16 & 0,60 & 0,06 & 0,03 & 0,11 \\
\hline Trema micrantha (L.) Blume & $\mathrm{PI}$ & 5,27 & 0,63 & 2,50 & 0,74 & 0,06 & 0,20 & 0,52 & 0,16 & 0,08 & 0,30 & 0,03 & 0,02 & 0,06 \\
\hline Morfoespécie 1 & NC & 5,27 & 0,63 & 2,50 & 0,74 & 0,06 & 0,18 & 0,51 & 0,14 & 0,07 & 0,26 & 0,03 & 0,01 & 0,05 \\
\hline Siparuna guianensis Aubl. & $\mathrm{SI}$ & 5,27 & 0,63 & 2,50 & 0,74 & 0,05 & 0,16 & 0,51 & 0,11 & 0,06 & 0,20 & 0,02 & 0,01 & 0,04 \\
\hline Maytenus sp. & NC & 5,27 & 0,63 & 2,50 & 0,74 & 0,04 & 0,13 & 0,50 & 0,11 & 0,06 & 0,20 & 0,02 & 0,01 & 0,04 \\
\hline Carpotroche brasiliensis (Raddi) A Gray & SI & 5,27 & 0,63 & 2,50 & 0,74 & 0,04 & 0,12 & 0,50 & 0,10 & 0,05 & 0,18 & 0,02 & 0,01 & 0,03 \\
\hline Guatteria ferruginea A.St.-Hil. & ST & 5,27 & 0,63 & 2,50 & 0,74 & 0,04 & 0,11 & 0,49 & 0,10 & 0,05 & 0,18 & 0,02 & 0,01 & 0,03 \\
\hline Micropholis crassipedicellata (Mart. \& Eichler) Pierre & $\mathrm{PI}$ & 5,27 & 0,63 & 2,50 & 0,74 & 0,04 & 0,11 & 0,49 & 0,11 & 0,05 & 0,20 & 0,02 & 0,01 & 0,04 \\
\hline Hyeronima alchorneoides Allemão & $\mathrm{PI}$ & 5,27 & 0,63 & 2,50 & 0,74 & 0,03 & 0,10 & 0,49 & 0,07 & 0,04 & 0,13 & 0,01 & 0,01 & 0,03 \\
\hline Tabernaemontana laeta Mart. & SI & 5,27 & 0,63 & 2,50 & 0,74 & 0,03 & 0,09 & 0,49 & 0,04 & 0,02 & 0,07 & 0,01 & 0,00 & 0,01 \\
\hline Myrocarpus frondosus Allemão & ST & 5,27 & 0,63 & 2,50 & 0,74 & 0,03 & 0,09 & 0,48 & 0,07 & 0,03 & 0,13 & 0,01 & 0,01 & 0,02 \\
\hline Morfoespécie 5 & NC & 5,27 & 0,63 & 2,50 & 0,74 & 0,03 & 0,08 & 0,48 & 0,06 & 0,03 & 0,11 & 0,01 & 0,01 & 0,02 \\
\hline Pterocarpus rohrii Vahl & SI & 5,27 & 0,63 & 2,50 & 0,74 & 0,03 & 0,08 & 0,48 & 0,12 & 0,06 & 0,22 & 0,02 & 0,01 & 0,04 \\
\hline Cariniana legalis (Mart.) Kuntze & C & 5,27 & 0,63 & 2,50 & 0,74 & 0,02 & 0,07 & 0,48 & 0,04 & 0,02 & 0,08 & 0,01 & 0,00 & 0,01 \\
\hline Zollernia glabra (Spreng.) Yakovlev & $\mathrm{SI}$ & 5,27 & 0,63 & 2,50 & 0,74 & 0,02 & 0,07 & 0,48 & 0,04 & 0,02 & 0,07 & 0,01 & 0,00 & 0,01 \\
\hline Couepia schottii Fritsch & NC & 5,27 & 0,63 & 2,50 & 0,74 & 0,02 & 0,07 & 0,48 & 0,05 & 0,03 & 0,10 & 0,01 & 0,01 & 0,02 \\
\hline Aloysia virgata (Ruiz \& Pav.) Juss. & $\mathrm{PI}$ & 5,27 & 0,63 & 2,50 & 0,74 & 0,02 & 0,06 & 0,48 & 0,05 & 0,03 & 0,10 & 0,01 & 0,00 & 0,02 \\
\hline Clarisia ilicifolia (Spreng.) Lanj. \& Rossberg & C & 5,27 & 0,63 & 2,50 & 0,74 & 0,02 & 0,06 & 0,47 & 0,03 & 0,02 & 0,06 & 0,01 & 0,00 & 0,01 \\
\hline Casearia oblongifolia Cambess. & NC & 5,27 & 0,63 & 2,50 & 0,74 & 0,02 & 0,06 & 0,47 & 0,04 & 0,02 & 0,07 & 0,01 & 0,00 & 0,01 \\
\hline Platymiscium floribundum Vogel & SI & 5,27 & 0,63 & 2,50 & 0,74 & 0,02 & 0,05 & 0,47 & 0,05 & 0,02 & 0,09 & 0,01 & 0,00 & 0,02 \\
\hline Balfourodendron riedelianum (Engl.) Engl. & C & 5,27 & 0,63 & 2,50 & 0,74 & 0,01 & 0,04 & 0,47 & 0,03 & 0,01 & 0,05 & 0,01 & 0,00 & 0,01 \\
\hline Protium heptaphyllum (Aubl.) Marchand & SI & 5,27 & 0,63 & 2,50 & 0,74 & 0,01 & 0,04 & 0,47 & 0,02 & 0,01 & 0,04 & 0,00 & 0,00 & 0,01 \\
\hline Andradea floribunda Allemão & ST & 5,27 & 0,63 & 2,50 & 0,74 & 0,01 & 0,04 & 0,47 & 0,02 & 0,01 & 0,04 & 0,00 & 0,00 & 0,01 \\
\hline Eriotheca pentaphylla (Vell. \& K.Schum.) A.Robyns & SI & 5,27 & 0,63 & 2,50 & 0,74 & 0,01 & 0,04 & 0,47 & 0,03 & 0,01 & 0,05 & 0,01 & 0,00 & 0,01 \\
\hline Gallesia integrifolia (Spreng.) Harms & ST & 5,27 & 0,63 & 2,50 & 0,74 & 0,01 & 0,03 & 0,47 & 0,02 & 0,01 & 0,03 & 0,00 & 0,00 & 0,01 \\
\hline
\end{tabular}

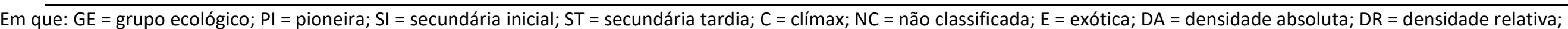

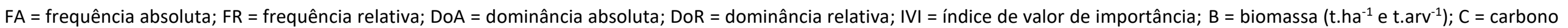

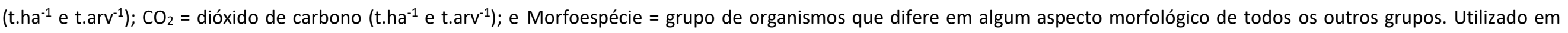
estudos ecológicos quando ainda não é possível a identificação da espécie. 
A espécie com maior densidade (68,49 plantas.ha $\left.{ }^{-1}\right)$ foi Artocarpus heterophyllus, espécie exótica, de grande facilidade de dispersão. A espécie nativa de maior densidade $\left(57,96\right.$ plantas.ha- $\left.{ }^{-1}\right)$ foi Actinostemon verticillatus (Klotzsch) Baill. Na Tabela 1 estão descritas todas as espécies, os parâmetros fitossociológicos classificados por IVI, o estoque de biomassa, carbono e $\mathrm{CO}_{2}$ totais e por indivíduo.

As 5 famílias mais representativas, em número de indivíduos foram, respectivamente, Moraceae (15,63\%), Euphorbiaceae (12,50\%), Meliaceae (8,13\%), Nyctaginaceae $(6,88 \%)$ e Sapindaceae $(5,63 \%)$. Juntas elas correspondem a $40,84 \%$ da biomassa total estimada. Já as 10 famílias com maior potencial de estoque de biomassa e, consequentemente carbono, estão dispostas na Figura 3 , sendo a família Meliaceae a de maior destaque $(23,46$ $\%)$.

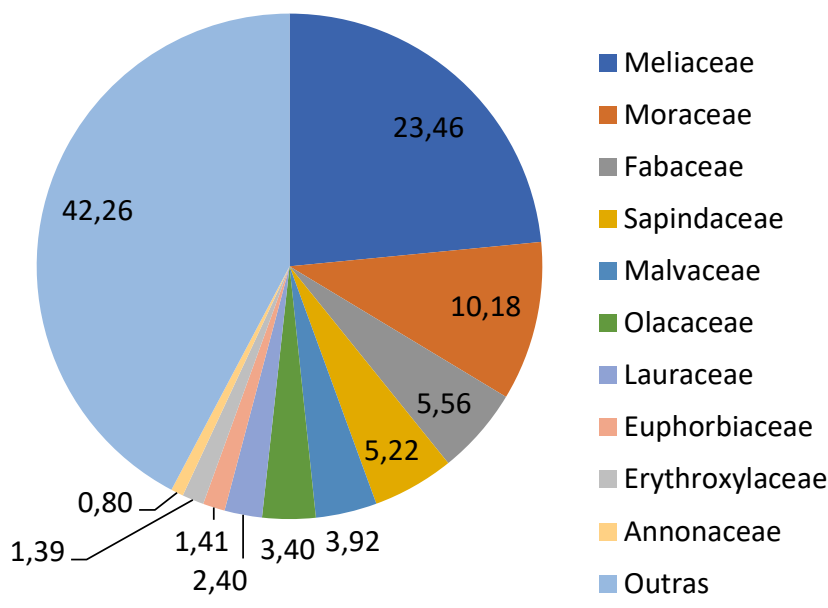

Figura 3. Famílias com maior estoque de biomassa em (\%), no PNMC, Paracambi, RJ.

Os estoques de biomassa e carbono estimados foram, respectivamente, 212,39 e 106,19 t.ha-1. A estratificação do estoque de biomassa por grupo ecológico (Tabela 2) demonstra que as espécies secundárias estocam as maiores quantidades (38,95\%). Isso é devido ao estágio de desenvolvimento intermediário da floresta, fase em que essas espécies ocorrem com maior densidade.

Dentre as espécies classificadas quanto ao grupo ecológico, o grupo das secundárias tardias é o de maior concentração de carbono por árvore e consequentemente o de maior potencial de fixação de $\mathrm{CO}_{2}$ (Tabela 2). Isso se deve ao diâmetro acentuado destes indivíduos na estimativa do VTCC por essa classe e consequentemente, corrobora com o maior poder de imobilização no material lenhoso da parte aérea.
Tabela 2. Estoque de biomassa, carbono, dióxido de carbono e percentual de carbono estratificado por grupo ecológico.

\begin{tabular}{ccccccc}
\hline $\mathrm{GE}$ & $\boldsymbol{V} \boldsymbol{T}_{c c}$ & $\boldsymbol{B}$ & $\boldsymbol{C}$ & $\mathrm{CO}_{2}$ & $\boldsymbol{C O}_{2 \text { (Árv.) }}$ & $\boldsymbol{C}(\%)$ \\
\hline PI & 0,17 & 31,54 & 15,77 & 57,82 & 10,35 & 15,97 \\
SI & 41,02 & 11,46 & 5,73 & 21,01 & 1,5 & 1,93 \\
ST & 176,26 & 49,85 & 24,92 & 91,39 & 3,59 & 37,02 \\
C & 69,44 & 0,11 & 0,05 & 0,19 & 0,04 & 0,39 \\
E & 18,69 & 20,91 & 10,45 & 38,33 & 0,51 & 17,91 \\
NC & 93,32 & 98,53 & 49,27 & 180,64 & 6,06 & 26,77 \\
\hline
\end{tabular}

Em que: $\mathrm{GE}=$ grupo ecológico; $V T_{c c}=$ volume total com casca $\left(\mathrm{m}^{3}\right.$.ha $\left.{ }^{-1}\right) . B=$ biomassa (t.ha-1); $C=$ carbono (t.ha-1 $\left.{ }^{-1}\right) ; \mathrm{CO}_{2}=$ dióxido de carbono equivalente por área $\left(\mathrm{t} . \mathrm{ha}^{-1}\right) ; \mathrm{CO}_{2(\hat{A} r v .)}=$ dióxido de carbono equivalente por árvore $\left(\operatorname{t}^{-a r v^{-1}}\right) ; C(\%)=$ carbono; em percentagem, $\mathrm{PI}=$ pioneira; $\mathrm{SI}=$ secundária inicial; $\mathrm{ST}=$ secundária tardia; $\mathrm{C}=$ clímax; $\mathrm{E}=$ exótica; $\mathrm{e} \mathrm{NC}=$ não classificada.

As dez espécies identificadas no PNMC com maior representatividade na imobilização do $\mathrm{CO}_{2}$ estão representadas na Figura 4, sendo Plathymenia reticulata Benth a que mais contribui. O potencial estocado e compreendido por toda a área amostral totaliza 3.893,81 toneladas de $\mathrm{CO}_{2}$ que deixam de estar na atmosfera.

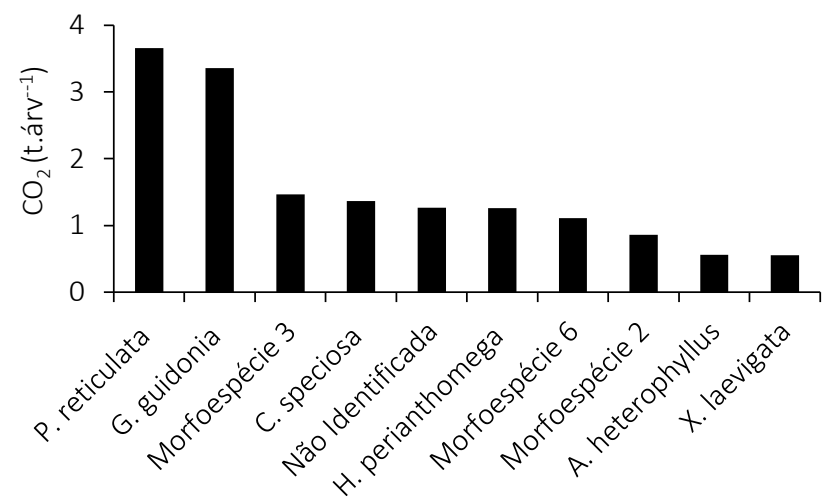

Figura 4. Espécies com maior estoque de biomassa (\%) no PNMC, Paracambi - RJ.

O estoque de carbono calculado nesse estudo foi muito similar ao encontrado por Weber et al. (2005), que verificaram cerca de $104 \mathrm{t}$ de carbono imobilizado em 1 ha de Floresta Ombrófila Mista, localizada em São João do Triunfo, PR. Porém, a área do referido trabalho já encontra-se em estágio de sucessão avançado, mesmo com a ocorrência de exploração seletiva no passado diferentemente desse estudo, que encontra-se em estágio de sucessão médio a avançado, mas dispõe de um longo período de regeneração natural, aumentando o 
potencial de estocar carbono ao longo do tempo. Além disso, essa similaridade de estimativas mesmo em distintos estágios de sucessão pode ser explicada pelas diferentes tipologias florestais abordadas entre os estudos e pela exploração seletiva de espécies com características favoráveis ao acúmulo de biomassa em sua madeira.

Ribeiro et al. (2009), utilizando a estimativa de volume do fuste sem casca e valores de densidade básica média encontradas na literatura para a determinação da biomassa em floresta madura, no município de Viçosa, MG, encontraram o valor de 164,13 t.ha-1 e 82,06 t.ha ${ }^{-1}$ para biomassa e carbono, respectivamente.

Em dois fragmentos florestais de Mata Atlântica no Parque Estadual do Desengano, município de Santa Maria Madalena, RJ, utilizando valores médios de densidade para espécies nativas e volume com casca diferenciado para classes de diâmetro distintas, verificou-se valores de 148,41 e 167,86 t.ha-1 ${ }^{-1}$ para biomassa total, considerando na estimativa galhos (13 e $10 \%$ do valor total das áreas) e folhas ( 5 e $4 \%$ do valor total das áreas) (CUNHA et al., 2009).

Diniz et al. (2015) estudaram duas tipologias florestais da Mata Atlântica no estado do Rio de Janeiro, floresta secundária em estágio médio (FSEM) e floresta secundária em estágio avançado (FSEA) de sucessão ecológica, ambas em Floresta Estacional Semidecidual Submontana. Os valores de biomassa e estoque de carbono encontrados para a FSEM, de 34,3 t.ha ${ }^{-1}$ e 15,4 t.ha ${ }^{-1}$, respectivamente, foram muito inferiores aos encontrados no presente trabalho, assim como para FSEA, com valores de 115,6 t.ha-1 e 52,0 t.ha ${ }^{-1}$, respectivamente. Tal inferioridade pode ser também observada em trabalho realizado por Amaro et al. (2013) em Floresta Estacional Semidecidual Montana em Minas Gerais, onde os valores estimados para biomassa e estoque de carbono foram de 134,83 t.ha ${ }^{-1}$ e 64,42 t.ha $^{-1}$, respectivamente.

As diferenças de estimativa dos parâmetros analisados podem ser decorrentes de inúmeros fatores, como as condições de cada área de estudo, o estágio de sucessão, a diferença de métodos de amostragem utilizados, histórico de uso e ocupação do solo local, qualidade do sítio, declividade, efeitos de borda e altitude e condições climáticas (WEBER et al., 2005). Além disso, as tipologias florestais em questão apresentam por essência variações em suas características intrínsecas à sua classificação que corroboram para uma discrepância entre os dados analisados.

\section{CONCLUSÕES}

Por meio do estudo realizado foi possível verificar a grande importância de Unidades de Conservação (UCs) para a manutenção da diversidade das florestas da Mata Atlântica e atenuação dos efeitos negativos das mudanças climáticas, pois viabiliza remoção significativa de gás carbônico da atmosfera, por meio da biomassa e o carbono estocados no estrato arbóreo.

A determinação do estoque de carbono (EC) por grupos ecológicos indica as espécies com maior potencial de fixação, sendo um indicador relevante nos estudos de recomposição florestal visando a venda de créditos de carbono. O grupo de maior destaque no EC é o das secundárias tardias e a espécie que mais estoca é Plathymenia reticulata Benth.

A imobilização de $\mathrm{CO}_{2}$ pelas UCs corresponde a uma alternativa de utilização de serviços ambientais, como o sequestro de carbono, de extrema importância para a manutenção dessas áreas, subsidiando projetos de Pagamentos de Serviços Ambientais e corroborando para a conservação da biodiversidade existente.

\section{AGRADECIMENTOS}

À Fundação Carlos Chagas Filho de Amparo à Pesquisa do Estado do Rio de Janeiro - FAPERJ, pelo subsídio a realização dessa pesquisa; e ao Parque Natural Municipal do Curió - PNMC, Paracambi, RJ, pela concessão da área de estudo.

\section{REFERÊNCIAS}

ALVARES, C.A. et al. Koppen's climate classification map for Brazil. Meteorologische Zeitschrift, v.22, n.6, p.711-728, 2014.

AMARAL, L.P. et al. Análise da distribuição espacial de biomassa e carbono arbóreo acima do solo em Floresta Ombrófila Mista. Ambiência, v.6, p.103-114, 2010.

AMARO, M.A. et al. Estoque volumétrico, de biomassa e de carbono em uma Floresta Estacional Semidecidual em Viçosa, Minas Gerais. Revista Árvore, v.37, n.5, p.849-857, 2013.

APG III. An update of the Angiosperm Phylogeny Group classification for the orders and families of flowering plants: APG III. Botanical Journal of the Linnean Society, v.161, p.105-121, 2009.

ARAÚJO, E.J.G. et al. Allometric models to biomass in restoration areas in the Atlantic rain forest. Floresta e Ambiente, v.25, n.1, e20160193, 2018.

BRASIL. Resolução CONAMA no 006, de 4 de maio de 1994. Diário Oficial da União, Brasília, 1994. Disponível em: 
http://www.mma.gov.br/port/conama/legiabre.cfm?codlegi=14 7

BROWN, S. et al. Biomass of tropical tree plantations and its implications for the global carbon budget. Canadian Journal of Forestry Research, v.16, p.390-394, 1986.

COTTAM, G.; CURTIS, J.T. The use of distance measures in phytosociological sampling. Ecology, v.37, n.3, p.451-460, 1956.

CUNHA, G.M. et al. Biomassa e estoque de carbono e nutrientes em florestas montanas da mata atlântica na região norte do estado do Rio de Janeiro. Revista Brasileira de Ciência do Solo, v.33, p.1175-1185, 2009.

CYSNEIROS, V.C. et al. Diversity, community structure and conservation status of an Atlantic Forest fragment in Rio de Janeiro State, Brazil. Biota Neotropica, v.15, n.2, e20140132, 2015.

DINIZ, A.R. et al. Biomassa, estoques de carbono e de nutrientes em estádios sucessionais da Floresta Atlântica, RJ. Revista Brasileira de Ciências Agrárias, v.10, n.3, p.443-451, 2015.

FRAGA, M.E. et al. Interação microrganismo, solo e flora como condutores de biodiversidade na Mata Atlântica. Acta Botânica Brasilica, v.26, n.4, p.857-865, 2012.

HASSLER, M.L. A importância das Unidades de Conservação no Brasil. Sociedade \& Natureza, v.17, n.33, p.79-89, 2005.

HOEN, H.; SOLBERG, B. Potencial and economic efficiency of cabon sequestration in Forest biomass through silvicultural management. Forest Science, v.40, n.3, p.429-451, 1994.

LIPINSKI, E.T. et al. Dinâmica da biomassa e carbono arbóreo entre 1995-2012 em Floresta Ombrófila Mista Montana. Floresta, v.47, n.2, p.197-206, 2017.

MOGNON, F. et al. Uma década de dinâmica da fixação de carbono na biomassa arbórea em Floresta Ombrófila Mista no sul do Paraná. Floresta, v.43, n.1, p.153-164, 2013.

MORAIS, V.A. et al. Carbon and biomass stocks in a fragment of cerradão in Minas Gerais state, Brazil. Cerne, v.19, n.2, p.237245, 2013.

MORAIS, V.A et al. Spatial and vertical distribution of litter and belowground carbon in a brazilian cerrado vegetation. Cerne, v.23, n.1, p.43-52, 2017.

MOREIRA-BURGER, D.; DELITTI, W.B.C. Fitomassa epigéa da mata ciliar do rio Mogi-Guaçu, Itapira - SP. Revista Brasileira de Botânica, v.22, n.3, p.429-435, 1999.

NICOLETTI, M.F. et al. Metodologia não destrutiva para quantificação do volume e biomassa do fuste em remanescente florestal. Nativa, v.3, n.4, p.287-291, 2015.

RIBEIRO, S.C. et al. Quantificação de biomassa e estimativa de estoque de carbono em uma floresta madura no município de Viçosa, Minas Gerais. Revista Árvore, v.33, n.5, p.917-926, 2009. em reflorestamento (estudo de caso: Mimosa scabrella Bentham, bracatinga). 2001. 86p. (Tese de doutorado).

SANQUETTA, C.R. et al. Relações individuais de biomassa e conteúdo de carbono em plantações de Araucaria angustifolia e Pinus taeda no sul do estado do Paraná, Brasil. Revista Acadêmica: ciências agrárias e ambientais, v.1, n.3, p.33-40, 2003.

SANTOS, R.C. et al. Estoques de volume, biomassa e carbono na madeira de espécies da Caatinga em Caicó, RN. Pesquisa Florestal Brasileira, v.36, n.85, p.1-7, 2016.

SCOLFORO, J.R. et al. Inventário florestal de Minas Gerais: equações de volume, peso de matéria seca e carbono para diferentes fisionomias da flora nativa. 22. ed. Lavras: UFLA, 2008.

SMMAP \& ITPA - Secretaria Municipal de Meio Ambiente de Paracambi \& Instituto Terra de Preservação Ambiental. Plano de manejo do Parque Natural Municipal do Curió de Paracambi. Paracambi, 2010.

SILVEIRA, P. Estimativa da biomassa e carbono acima do solo em um fragmento de Floresta Ombrófila Densa utilizando o método da derivação do volume comercial. Floresta, v.40, n.4, p.789800, 2010.

SOUZA, A.L. et al. Estoque e crescimento em volume, biomassa, carbono e dióxido de carbono em Floresta Estacional Semidecidual. Revista Árvore, v.35, n.6, p. 1277-1285, 2011.

TEIXEIRA, G.M. et al. Regeneração de floresta atlântica sob níveis diferenciados de perturbação antrópica: implicações para restauração. Scientia Forestalis, v.42, n.104, p543-554, 2014.

WATZLAWICK, L.F. et al. Estoque de biomassa e carbono na Floresta Ombrófila Mista Montana Paraná. Scientia Forestalis, v.40, n.95, p.353-362, 2012.

WEBER, K.S. et al. Variação volumétrica e distribuição espacial do estoque de carbono em floresta ombrófila mista. Revista Acadêmica, v.3, n.2, p.77-85, 2005. 